\title{
Fusobacterium russii
}

National Cancer Institute

\section{Source}

National Cancer Institute. Fusobacterium russii. NCI Thesaurus. Code C86405.

A species of anaerobic, Gram-neg ative, fusiform rod shaped bacteria in the phylum Fusobacteria. This species is lipase negative, does not hydrolyze esculin, does not ferment carbohydrates, does not grow in bile, does not produce propionate from lactate or threonine, but does have phosphate activity. F. russii has been isolated from both animal and human feces and is associated with human infections particularly those caused by cat and dog bites. 